\title{
ARTIGO 8
}

\section{PREDISPOSIÇÃO PARA SÍNDROME DE BURNOUT NA EQUIPE DE ENFERMAGEM DO SERVIÇO DE ATENDIMENTO MÓVEL DE URGÊNCIA}

Franciana Gabaglia da Silva ${ }^{1}$, Abigail de Paulo Andrade ${ }^{1}$, Keila Maria de Azevedo Ponte ${ }^{1}$, Verena Emmanuelle Soares Ferreira ${ }^{2}$, Beatriz da Silva Sousa ${ }^{1}$, Kauanny Gomes Gonçalves ${ }^{1}$

Objetivo: conhecer a predisposição para a Síndrome de Burnout na equipe de Enfermagem do Serviço de Atendimento Móvel de Urgência (SAMU). Metodologia: pesquisa exploratória, descritiva realizada com 08 enfermeiros e 11 técnicos de enfermagem do SAMU de Sobral-Ceará. Utilizou-se o Questionário para Identificação Preliminar do Burnout de Chafic Jbeli, o Maslach Burnout Inventary (MBI) e um questionário sócio demográfico e profissional. Projeto aprovado pelo Comitê de Ética em Pesquisa com Seres Humanos. Resultados: a maioria dos profissionais apresenta nível baixo para exaustão emocional; nível moderado para despersonalização; e nível alto de reduzida realização profissional, evidenciando predisposição à Síndrome de Burnout. Conclusão: a equipe de enfermagem do SAMU tem predisposição a Síndrome de Burnout, sendo primordial o enfrentamento dos fatores estressores da Enfermagem e adoção de medidas preventivas para reduzir o estresse laboral.

Descritores: Enfermagem; Estresse ocupacional; Assistência Pré-Hospitalar.

\section{PREDISPOSITION FOR BURNOUT SYNDROME IN THE NURSING TEAM OF THE URGENT MOBILE CARE SERVICES}

Objective: to know the predisposition for Burnout Syndrome in the nursing team of the Mobile Emergency Care Service (SAMU). Methodology: exploratory, descriptive study performed with 08 nurses and 11 nursing technicians from SAMU of SobralCeará. The Chafic Jbeli Burnout Preliminary Identification Questionnaire, the Maslach Burnout Inventary (MBI) and a sociodemographic and professional questionnaire were used. Project approved by the Committee of Ethics in Research with Human Beings. Results: most professionals present low level of emotional exhaustion; moderate level for depersonalization; and high level of reduced professional achievement, evidencing a predisposition to Burnout Syndrome. Conclusion: the SAMU nursing team is predisposed to Burnout Syndrome, and it is essential to address the stressors of Nursing and to adopt preventive measures to reduce work stress.

Descriptors: Nursing; Occupational stress; Prehospital Care.

\section{PREDISPOSICIÓN PARA SÍNDROME DE BURNOUT EN EL EQUIPO DE ENFERMERÍA DEL SERVICIO DE ATENCIÓN MÓVIL DE URGENCIA}

Objetivo: conocer la predisposición para el Síndrome de Burnout en el equipo de Enfermería del Servicio de Atención Móvil de Urgencia (SAMU). Metodología: investigación exploratoria, descriptiva realizada con 08 enfermeros y ll técnicos de enfermería del SAMU de Sobral-Ceará. Se utilizó el Cuestionario para Identificación Preliminar del Burnout de Chafic Jbeli, el Maslach Burnout Inventario (MBI) y un cuestionario socio demográfico y profesional. Proyecto aprobado por el Comité de Ética en Investigación con Seres Humanos. Resultados: la mayoría de los profesionales presentan un nivel bajo para el agotamiento emocional; nivel moderado para despersonalización; y nivel alto de reducida realización profesional, evidenciando predisposición al Sindrome de Burnout. Conclusión: el equipo de enfermería del SAMU tiene predisposición al Síndrome de Burnout, siendo primordial el enfrentamiento de los factores estresores de la Enfermería y adopción de medidas preventivas para reducir el estrés laboral.

Descriptores: Enfermeria; Estrés ocupacional; Asistencia Pre-Hospitalaria.

1Universidade Estadual Vale do Acaraú-UVA.Acaraú.CE.

2Secretaria Municipal de Saúde de Sobral,CE.

Autor correpondente: Franciana Gabaglia da Silva. E-mail: franciana_gabaglia@hotmail.com 


\section{INTRODUÇÃO}

A sindrome de Burnout é uma psicopatologia ocupacional resultante de três dimensões: a exaustão emocional que gera exteriorização das sensações de estar além dos limites; a despersonalização que gera atitudes negativas e de cinismo; e a reduzida realização pessoal que gera avaliações negativas do individuo quanto ao trabalho e perspectivas futuras em seu labor'.

O Burnout pode acometer qualquer profissional independente da área de ocupação, porém, os profissionais expostos ao sofrimento alheio como a Enfermagem, tendem a ter maior risco para desenvolver o Burnout ${ }^{2}$.

Neste sentido, os profissionais do Serviço de Atendimento Móvel de Urgência (SAMU) estão submetidos continuamente a riscos, tanto a nível físico como psíquico por tratar de um ambiente permeado de pressão e imprevisibilidade, além de lidarem com o risco iminente de morte. Assim, dentre os principais riscos ocupacionais que estes profissionais estão submetidos citam-se perda auditiva induzida por ruídos devido à sirene da ambulância; acidentes de trânsito; lesões por esforços repetitivos e distúrbios osteomusculares relacionados ao trabalho com o levantamento contínuo de macas; exposição a agentes biológicos como sangue, fluidos e estresse nas ocorrências ${ }^{3.4}$.

No Brasil, a Previdência Social afirma que, em 2012 a 2016, foram afastados 668.927 individuos por transtornos mentais e comportamentais, sendo a terceira causa de incapacidade para o trabalho, sendo 34.511 diagnosticados com Síndrome de Burnout ${ }^{5}$

A enfermagem é avaliada como a quarta ocupação mais desgastante no setor público segundo a Health Education Authority. E dentre as áreas da enfermagem, o âmbito do pré-hospitalar expõe os profissionais a condições de riscos, tornando-os mais vulneráveis. No SAMU Fortaleza, a enfermagem classifica a ocupação como moderadamente estressante e muito estressante; e no SAMU de Maceió e Arapiraca-AL evidenciou-se que $76,3 \%$ dos enfermeiros apresentavam Síndrome de Burnout ${ }^{6}$.

Considerando o papel da enfermagem no SAMU, tendo como eixo norteador a qualidade de vida destes trabalhadores, esta pesquisa teve como questão norteadora: Qual a predisposição da Síndrome de Burnout na equipe de enfermagem do SAMU?

Na perspectiva de superar as barreiras fisicas e estruturais do sistema, respeitando a realidade específica do serviço, objetivou-se conhecer a predisposição da Síndrome de Burnout na equipe de enfermagem do SAMU.

\section{METODOLOGIA}

\section{Tipo de estudo}

Trata-se de uma pesquisa exploratória e descritiva quantitativa.

\section{Participantes da pesquisa}

A população foi de 20 indivíduos, sendo sete enfermeiros e onze técnicos de enfermagem. Os critérios de inclusão foram ser profissionais de enfermagem, com vínculo empregatício e atuante no serviço supracitado durante a vigência da pesquisa. E como critério de exclusão considerou-se a recusa de participar da pesquisa, as férias, as licenças médicas e/ou a licença maternidade. Deste modo, dos vinte profissionais do serviço, participaram dezoito.

\section{Local do estudo}

Estudo realizado no Serviço de Atendimento Médico de Urgência na cidade de Sobral no Ceará.

\section{Coleta dos dados}

O questionário foi individual e aleatório, no horário disponivel dos profissionais entre as ocorrências do plantão e não prejudicou a rotina do serviço. O local das entrevistas variou de acordo com a disponibilidade dos espaços oferecidos na unidade. A coleta de dados foi em Setembro de 2016, após a assinatura do Termo de Consentimento Livre e Esclarecido $(T C L E)^{7}$ foi utilizado o questionário sociodemográfico e profissional com questões fechadas elaborado pelas autoras; - Questionário para Identificação Preliminar da Burnout. elaborado e adaptado por Chafic Jbeliं; e as variantes do Maslach Burnout Inventary (MBI) ${ }^{8}$.

O Questionário para Identificação Preliminar da Burnout foi composto com vinte alternativas assim distribuídas: nove referentes à Exaustão Emocional (Questões 1, 2, 3, 6, 10, 11, 14 . 19, 20); quatro referentes à Despersonalização (Questões 5 , 12, 15, 18); sete referentes à Reduzida Realização Profissional (Questões 4, 7, 8, 9, 13, 16, 17); em que os voluntários devem responder de acordo com o conteúdo sugerido pelo item. Quanto ao número de questões o Questionário para Identificação Preliminar da Burnout assinaladas nas categorias: de $\mathrm{O}$ a 20 pontos - nenhum indício de Burnout: de 21 a 40 pontos - possibilidade de desenvolver Burnout de 41 a 60 - fase inicial da Burnout; de 61 a 80 pontos - a Burnout começa a se instalar; de 81 a 100 - fase considerada da Burnout.

\section{Procedimentos de análise dos dados}

O instrumento respondido foi codificado e digitado no Excel 2010. Para análise dos dados relativos ao instrumento de MBI, realizou-se a somatória de cada dimensão (exaustão emocional, despersonalização e reduzida realização 
profissional).

Procedimentos éticos

A pesquisa foi aprovada pelo Comitê de Ética em Pesquisa da Universidade Estadual Vale do Acaraú com o Parecer Consubstanciado o no 1.692.062/2016 e ressalta-se que foram seguidos os cuidados éticos que preceituam a Resolução 510/16, do Conselho Nacional de Saúde(7).

\section{RESULTADOS}

A amostra utilizada no presente estudo foi composta por 18 profissionais de enfermagem atuantes no SAMUSobral. A Tabela 1 demonstra o perfil sociodemográfico dos participantes.

Tabela 1 - Perfil sociodemográfico dos profissionais de enfermagem do SAMU, segundo a idade, sexo, estado civil, profissão, renda familiar no SAMU. Sobral-Ceará, 2016.

\begin{tabular}{lcc}
\hline VARIÁVEIS & $\mathbf{n}$ & $\%$ \\
\hline Idade & & \\
$20-29$ & 08 & 44,4 \\
$30-39$ & 04 & 22,2 \\
$40-49$ & 03 & 16,7 \\
50 ou mais & 03 & 16,7 \\
Sexo & & \\
Masculino & 11 & 61,1 \\
Feminino & 07 & 38,9 \\
Estado Civil & & \\
Solteiro(a) & 12 & 66,7 \\
Casado(a) & 06 & 33,3 \\
\hline Total & 18 & 100 \\
\hline
\end{tabular}

Na Tabela l, observou-se que oito profissionais estavam na faixa etária entre 20 a 29 anos de idade; onze profissionais do sexo masculino; e doze eram solteiros.

Após o perfil sociodemográfico foi avaliado o perfil ocupacional dos profissionais apresentados na Tabela 2.

Tabela 2 - Perfil profissional dos profissionais de enfermagem do SAMU, segundo profissão, renda familiar, tempo de serviço prestado, carga horária semanal na instituição e carga horária semanal no SAMU e em outras instituições. Sobral-Ceará, 2016.

\begin{tabular}{lcc}
\hline VARIÁVEIS & $\mathbf{n}$ & $\%$ \\
\hline Profissão & & \\
Técnico de Enfermagem & 11 & 61,1 \\
Enfermeiro & 07 & 38,9 \\
Renda Familiar & & \\
$03-04$ salários-mínimos & 08 & 44,4 \\
$01-02$ salários-mínimos & 06 & 33,3 \\
05 ou mais salários-minimos & 04 & 22,3 \\
Tempo de Serviço Prestado no SAMU & & \\
$10-11$ anos & 07 & 38,9 \\
$01-02$ anos & 05 & 27,8 \\
$03-04$ anos & 05 & 27,8 \\
$05-06$ anos & 01 & 5,6 \\
Carga horária semanal no SAMU e em outras instituições & & \\
$81-100$ horas/semanais & 09 & 50,0 \\
$40-60$ horas/semanais & 04 & 22,3 \\
$61-80$ horas/semanais & 04 & 22,3 \\
Mais que 100 horas/semanais & 01 & 5,6 \\
\hline Total & 18 & 100 \\
\hline
\end{tabular}

Enferm. Foco 2019; 10 (1): 40-45
Quanto às categorias profissionais, a prevalência foi de onze técnicos de enfermagem, com renda familiar de 3 a 4 salários mínimos e sete profissionais com tempo de serviço prestado no SAMU entre 10 a 11 anos. A carga horária dos técnicos de enfermagem era de 44 horas e do enfermeiro 24 horas semanais, no entanto, devido à jornada dupla, 09 dos profissionais fazem a carga horária semanal entre SAMU e outras instituições de 81 a 100 horas semanais

Tabela 3 - Dimensões do Maslach Burnout Inventory, segundo a exaustão emocional, a despersonalização e a reduzida realização pessoal entre os profissionais de enfermagem do SAMU. Sobral-Ceará, 2016.

\begin{tabular}{lcccc}
\hline VARIÁVEIS & \multicolumn{3}{c}{ ENFERMEIROS } & \multicolumn{2}{c}{ TÉCNICOS DE } \\
& n & $\%$ & ENFERMAGEM \\
& n & \% \\
\hline Exaustão Emocional & & & & \\
$\quad$ Baixo & 04 & 57,1 & 04 & 36,4 \\
$\quad$ Moderado & 02 & 28,6 & 05 & 45,4 \\
$\quad$ Alto & 01 & 14,3 & 02 & 18,2 \\
$\quad$ Total & 07 & 100 & 11 & 100 \\
Despersonalização & & & & \\
$\quad$ Baixo & 03 & 42,8 & - & - \\
$\quad \begin{array}{l}\text { Moderado } \\
\quad \text { Alto }\end{array}$ & 04 & 57,2 & 10 & 90,9 \\
$\quad$ Total & - & - & 01 & 9,1 \\
$\quad \begin{array}{l}\text { Reduzida Realização Pessoal } \\
\quad \text { Baixo }\end{array}$ & 07 & 100 & 11 & 100 \\
$\quad \begin{array}{l}\text { Moderado } \\
\text { Alto }\end{array}$ & - & - & - & - \\
\hline Total & - & - & - & - \\
\hline & 07 & 100 & 11 & 100 \\
\hline & 07 & 100 & 11 & 100 \\
\hline
\end{tabular}

$\mathrm{Na}$ Tabela 3 verificou-se que entre os enfermeiros, quatro profissionais tinham nível baixo de exaustão emocional, quatro com nível moderado de despersonalização e sete no nivel alto de reduzida realização profissional; enquanto os técnicos de enfermagem, cinco tinham nível moderado de exaustão emocional, dez profissionais no nível moderado de despersonalização e onze no nível alto de reduzida realização profissional.

Tabela 4 - Perfil dos Resultados associados ao Questionário Preliminar de Identificação do Burnout entre os profissionais de enfermagem do SAMU. Sobral-Ceará, 2016.

\begin{tabular}{lcccc}
\hline VARIÁVEIS & \multicolumn{2}{c}{ ENFERMEIROS } & \multicolumn{2}{c}{ TÉCNICOS DE } \\
& $\mathbf{n}$ & $\%$ & $\mathbf{n}$ & $\%$ \\
\hline Nenhum indício & - & - & - & - \\
Possibilidade de desenvolver & 03 & 42,8 & 01 & 9,1 \\
Fase inicial & 02 & 28,6 & 09 & 81,8 \\
Fase de instalação & 02 & 28,6 & & 9,1 \\
Fase considerável & - & - & 01 & - \\
\hline Total & 07 & 100 & 11 & 100 \\
\hline
\end{tabular}

O Questionário Preliminar de Identificação do Burnout identificou uma classificação segundo a pontuação obtida 
por escores. Na Tabela 4 destaca-se que três enfermeiros apresentam sintomas correspondentes à possibilidade de desenvolver Burnout; e nove técnicos de enfermagem apresentam sintomas correspondentes à fase inicial do Burnout.

\section{DISCUSSÃO}

O estudo mostrou que os profissionais de enfermagem do SAMU são predominantemente jovens do sexo masculino e solteiros. Porém o perfil pessoal não é por si só desencadeante da Síndrome de Burnout, apenas facilitador ou não para o desenvolvimento do quadro. Estudos com o mesmo grupo em locais diferentes, este resultado foi similar; no SAMU Picos/Piauí, entre médicos, enfermeiros, técnicos e auxiliares de enfermagem e condutores foram $75 \%$ eram do sexo masculino, 43,8\% na faixa etária entre e 20 até 30 anos, 25\% enfermeiros, $84,4 \%$ trabalhavam no SAMU de 1 a 5 anos, $37,5 \%$ trabalhavam mais de 60 horas semanais; enquanto no SAMU - IGSS/Peru, entre médicos, enfermeiros e condutores foram $63,9 \%$ eram do sexo masculino, 57,6\% na faixa etária entre 31 a 40 anos, 32,9\% enfermeiros ${ }^{9-10}$

As dimensões da Síndrome de Burnout foram comparadas: Exaustão Emocional, Despersonalização e Reduzida Realização Profissional segundo os subníveis baixo, moderado e alto. Os escores da sub-escala da sindrome de Burnout para Exaustão Emocional são: baixo (0-16 pontos), moderado (1726 pontos) e alto (27 ou mais pontos); na Despersonalização são: baixo (0-6 pontos), moderado (7-12 pontos) e alto (13 ou mais pontos); e na Reduzida Realização Profissional são: baixo (39 ou mais pontos), moderado (32-38 pontos) e alto (0-31 pontos) ${ }^{8}$.

Com relação à prevalência da Síndrome de Burnout foi observado que todos os profissionais estudados já possuiam sintomas para uma das três dimensões da sindrome. A reduzida realização profissional em altos níveis pode gerar sensação de incapacidade, baixa autoestima, desmotivação e infelicidade no trabalho, afetando até a habilidade e a destreza, quando associado a sentimentos de insensibilidade em relação aos clientes ou colegas de trabalho, e frustação pode ser um desencadeador da Síndrome de Burnout.

Tratando-se das dimensões do Burnout, no SAMU - Picos/ Piauí foram encontrados resultados semelhantes, sendo $87,5 \%$ dos enfermeiros com nível baixo a moderado, enquanto $66,7 \%$ dos técnicos de enfermagem com reduzida realização profissional em nivel alto(8). No SAMU - IGSS/Peru foram $67,3 \%$ dos enfermeiros com presença de estresse laboral'

Inúmeros fatores podem estar relacionados ao desenvolvimento da Síndrome de Burnout no SAMU, haja vista que os profissionais atuantes na zona urbana enfrentam o trânsito caótico da cidade, assim como a má sinalização e os buracos em ruas; seguidas do trabalho em áreas de risco e do complicado relacionamento com a população; além do relacionamento com a equipe e a chefia.

Esta pesquisa mostrou que a maioria dos profissionais está situada na fase inicial da Síndrome de Burnout. Neste sentido, esta informação é relevante, corroborando com o fato dos sintomas da fase inicial do Burnout poderem confundir-se com sintomas de outros distúrbios mentais; além de afetar na qualidade da assistência prestada ao paciente.

A fase inicial pode ser confundida com a depressão, pois apresenta agressividade, isolamento, mudança de humor, irritabilidade, dificuldade de concentração, falha da memória, ansiedade, tristeza, pessimismo, baixa autoestima e ausência no trabalho"l. Os profissionais de enfermagem do SAMU - Sobral/CE ainda não desenvolveram estratégias de adaptação aos fatores estressantes da rotina de trabalho, sendo o trabalho considerado um fator predisponente ao adoecimento ocupacional.

Outro estudo com os profissionais de enfermagem do SAMU de uma cidade de médio porte da região norte catarinense, aponta que os profissionais entrevistados estão na fase inicial da Síndrome de Burnout ${ }^{12}$

O adoecimento do profissional com a Síndrome de Burnout gera repercussões negativas que pode ser entendida no déficit da qualidade da assistência prestada à população além de sentimentos de agressividade e desprezo com a equipe, prejudicando a vida pessoal, social e ocupacional do indivíduo.

Quando os trabalhadores sofrem desgaste físico e emocional, não são os únicos responsáveis pela fadiga, raiva e pela atitude de indiferença que adotam. Tal desgaste é sinal de uma disfunção importante no ambiente das organizações e, portanto, revela mais sobre o local de trabalho do que sobre os que nele trabalham ${ }^{13}$. Haja visto que as condições de trabalho na assistência pré-hospitalar são constituídas por ambientes hostis para atuação da equipe, demandas psicológicas intensas, sobrecarga ocupacional e recursos estruturais deficientes ${ }^{14}$

Não é vasta a relação da Síndrome Burnout e sua prevalência na literatura brasileira. Sendo apontada em 26\% da amostra de uma pesquisa que a classe de profissionais apresentou exaustão pelo trabalho. E que, nesta perspectiva, algumas empresas tratam a Síndrome como sendo um problema particular do trabalhador ${ }^{15}$

O Burnout é uma sindrome ocasionada por fatores biopsicossociais associados ao ambiente de trabalho, por si só o perfil individual não é desencadeador, o que a torna um problema de saúde pública e intimamente ligada à gestão organizacional dos serviços de trabalho. 
Limitações do estudo

Quanto às limitações deste estudo, houve amostra insuficiente diante do total de enfermeiros atuantes no serviço, dessa forma não permite a generalização dos resultados.

\section{Contribuições para a prática}

Este estudo pretende suscitar novas reflexões acerca da Sindrome de Burnout, principalmente sobre o impacto desta sindrome na organização do serviço, na vida dos profissionais e na satisfação dos usuários atendidos; bem como a busca de novas estratégias para prevenção e para acompanhamento longitudinal dos profissionais afetados pela sindrome, assim como a criação de novas atitudes para enfrentamento da Síndrome de Burnout.

\section{CONCLUSÃO}

Este estudo permitiu demonstrar a predisposição da Síndrome de Burnout na equipe de enfermagem do SAMU. Os profissionais apresentaram mais de duas dimensões em niveis altos para a Síndrome de Burnout associados à sintomatologia correspondente as fases do Burnout.

Quanto às dimensões da Síndrome de Burnout, os profissionais de enfermagem possuem nivel baixo para exaustão emocional; nivel moderado para despersonalização; e nivel alto para reduzida realização profissional. Dentro dos limites do Inventário de Burnout de Maslach, constatou-se que os enfermeiros possuem a possibilidade de desenvolver a Síndrome de Burnout; enquanto os técnicos de enfermagem vivenciam a fase inicial da sindrome.

A pressão psicológica, a agilidade e a rapidez exigidas no atendimento pré-hospitalar móvel geram um ambiente propicio ao desenvolvimento de doenças. Embora a Síndrome de Burnout seja um diagnostico clinico medico e feito de forma individual, os resultados obtidos neste estudo podem ser considerados como alerta para a instituição em relação ao adoecimento dos profissionais de enfermagem e um risco para os demais profissionais de saúde.

A investigação da predisposição da Síndrome de Burnout gera o repensar das ações acerca da assistência prestada aos usuários e das relações interpessoais, embora não tenha sido realizada intervenção, os resultados buscam gerar o pensamento critico nos profissionais acerca do processo de trabalho como agente adoecedor.

O enfrentamento da Síndrome de Burnout pode acontecer com a redução dos fatores estressores da enfermagem como a melhoria das condições de trabalho; quadro de funcionário de acordo com a necessidade do serviço; apoio estratégico de outras instituições como a policia civil e o corpo de bombeiros em ocorrências de risco. Portanto, a escuta qualificada e o acolhimento das necessidades dos profissionais pode ocasionar novas medidas na gestão do serviço, elucidando potenciais problemas desencadeadores do Burnout, o qual repercute negativamente na qualidade de vida e no desempenho laboral destes profissionais.

\section{Contribuição dos autores}

Concepção e desenho, análise e interpretação dos dados, redação do artigo, revisão crítica, revisão final: Franciana Gabaglia da Silva, Abigail de Paulo Andrade, Keila Maria de Azevedo Ponte, Verena Emmanuelle Soares Ferreira, Beatriz da Silva Sousa, Kauanny Gomes Gonçalves. 


\section{REFERÊNCIAS}

1. Medeiros-Costa ME, Maciel RH, Rêgo DP do, Lima LL de, Silva MEP da, Freitas JG. Occupational Burnout Syndrome in the nursing context: an integrative literature review. Rev da Esc Enferm da USP [Internet] 2017 [cited 2017 Dez 11];51:1-12. Available from: http://www.scielo.br/ scielo.php?script=sci_arttextEpid=S0080-623420170001008018lng= en\&tlng=en

2. Sousa AQ, Barros AG, Dias ACS, Santos VEP. Perspetivas de sindrome de Burnout na Equipe de Enfermagem da Unidade de Terapia Intensiva. Rev Pesqui Cuid é Fundam [Internet]. 2012 [cited 2017 Dez 11];4(3):2672-8. Available from: https://dialnet.unirioja.es/descarga/ articulo/5090847.pdf

3. Angelim RC de M, Rocha GS de A. Scientific production about the working conditions of nursing in emergency and urgent services. Rev Pesqui Cuid é Fundam Online [Internet]. 2016 [cited 2017 Dez 11]:8(1):3845. Available from: http://seer.unirio.br/ index.php/ cuidadofundamental/article/view/4473

4. Leite HDCS, Carvalho MTR de, Cariman SL da S, Araújo ER de M, Silva NC, Carvalho A de O. Risco Ocupacional entre Profissionais de Saúde do Serviço de Atendimento Móvel de Urgência - SAMU. Enferm em Foco [Internet]. 2016 [cited 2017 Dez 11];7(3/4):31-5. Available from: http://revista.portalcofen.gov.br/index.php/enfermagem/article/ view/912/342

5. Brasil. Ministério da Fazenda. Adoecimento mental e trabalho: a concessão de beneficios por incapacidade relacionados a transtornos mentais e comportamentais entre 2012 e 2016 / $1^{\circ}$ Boletim quadrimestral sobre beneficios por incapacidade [Internet]. 2017 [cited 2017 Dez 11]. Available from: http://www.previdencia.gov.br/wpcontent/uploads/2017/04/1\%C 2\%BA-boletim-quadrimestral.pdf

6. Martins AM de C, Santos VV, Mendonça IO, Silva DP da, Oliveira DM. Sindrome de burnout em profissionais da enfermagem do atendimento pré-hospitalar. Ciências Biológicas e de Saúde Unit [Internet]. 2017 [cited 2018 Apr 29];4(2):101-114. Available from: https://periodicos.set. edu.br/index.php/cadernobiologicas/article/viewFile/4595/2497

7. Brasil. Resolução n. 510, de 07 de abril de 2016. Aprova as diretrizes e normas regulamentadoras de pesquisas envolvendo seres humanos [Internet]. Diário Oficial da União: 13 jun 2016 [cited 2016 Mar 07]. Available from: http://conselho.saude.gov.br/ resolucoes/2016/ Reso510.pdf

8. Hoppen CMS, Kissmann N, Chinelato JR, Coelho VP, Wenczenovicz C, Nunes FCL, et al. High prevalence of burnout syndrome among intensivists of the city of Porto Alegre. Rev Bras Ter Intensiva [Internet]. 2017 [cited 2017 Dez 11];29(1):115-20. Available from: http:// www.gnresearch.org/doi/10.5935/0103-507X.20170017

9. Luz LM, Torres RRB, Sarmento KMV de Q, Sales JMR, Farias KN, Marques MB. Sindrome de burnout em profissionais do serviço de atendimento móvel de urgência. Rev Pesqui Cuid é Fundam Online [Internet]. 2017 [cited 2017 Dez 11]:9(1):238-46. Available from: http:// seer.unirio.br/index.php/cuidadofundamental/article/view/5400

10. Maura SVK, Amalia LPR, Hernán SVM. Prevalencia de estrés laboral en el personal asistencial prehospitalario del programa de sistema de atencion móvil de urgencias -Instituto de Gestión de Servicios de Salud. Rev enferm Hered [Internet]. 2015 [cited 2017 Dez 11];8(2):11622. Available from: http://www.upch.edu.pe/vrinve/dugic/ revistas/ index.php/RENH/article/view/2691/2567

11. Carvalhais FR, Aguilar AMM, Mendonça RL, Ottano C. Frequência da sindrome de Burnout em uma Unidade de Terapia Intensiva: uma perspectiva multiprofissional. Rev Prev Infec e Saúde [Internet]. 2015 [cited 2017 Dez 11];1(4):1-10. Available from: http://www.ojs.ufpi.br/ index.php/nupcis/article/view/4271/pdf

12. Reinert T, Bertoldi VS, Torres MS. Sindrome de Burnout em uma Perspectiva Avaliativa Aplicada em Profissionais de Serviço Móvel de Urgência. Psicologado: Revista Eletrônica Escritos Psicanalíticos [Internet]. 2016 [cited 2017 Jun 17]; 9(7). Available from: https:// psicologado.com/psicopatologia/saude-mental/sindromede-bournout-em-uma-perspectiva-avaliativa-aplicada-emprofissionais-de-servico-movel-de-urgencia

13. Oliveira LO de, Oliveira SMK de. A Sindrome de Burnout nas Organizações. Rev Maiêutica [Internet]. 2016 [cited 2017 Dez 11]:4(1):1726. Available from: https://publicacao.uniasselvi.com. br/index.php/ GESTAO_EaD/article/view/1589/718

14. Dal Pai D, Lima MAD da S, Abreu KP, Zucatti PB, Lautert L. Equipes e condições de trabalho nos serviços de atendimento pré-hospitalar móvel: revisão integrativa. Rev Eletr Enf [Internet]. 2015 [cited 2017 Dez 11]:17(4):1-12. Available from: http://revistas.ufg.emnuvens.com. br/fen/article/view/31522

15. Cândido J, Souza LR. Sindrome de Burnout: as novas formas de trabalho que adoecem. Psicologia [Internet]. 2017[cited 2018 May 04];1 (1):1-12. Available from: http://www.psicologia.pt/artigos/ver_artigo. php?sindrome-de-burnout-as-novas-formas-de-trabalho-queadoecem8codigo=A1054Earea $=\mathrm{d} 8$ 